\title{
Mucormycosis in renal transplant recipients: review of 174 reported cases
}

\author{
Yan Song ${ }^{1}$, Jianjun Qiao ${ }^{2}$, Gaffi Giovanni ${ }^{3}$, Guangjun Liu${ }^{1}$, Hao Yang ${ }^{1}$, Jianyong Wu and Jianghua Chen ${ }^{*}$
}

\begin{abstract}
Background: Mucormycosis is a highly lethal fungal infection especially in immunocompromised individuals.

Methods: In order to review the epidemiology, diagnosis, and treatment of mucormycosis in renal transplant recipients we searched publications of mucormycosis cases in renal transplant recipients in PUBMED database up to December 2015.

Results: A total of 174 cases in renal transplant recipients were included in this review. Most of the cases (76\%) were male. Major underlying diseases were diabetes mellitus (43.1\%). Rhinocerebral was the most common site of infection (33.3\%). Rhizopus species was the most frequent fungus (59.1\%) in patients with pathogen identified to species level. The mortality rates of disseminated mucormycosis (76.0\%) and graft renal (55.6\%) were higher than infection in other sites. The overall survival in patients received surgical debridement combined with amphotericin B/posaconazole (70.2\%) was higher than those who received antifungal therapy alone (32.4\%), surgery alone (36.4\%) or without therapy $(0 \%)(p<0.001)$. The overall survivals in patients receiving posaconazole and lipid amphoterincin B were higher than that receiving deoxycholate formulation ( $92.3 \%$ and $73.4 \%$ vs $47.4 \%$ ).

Conclusions: Mucormycosis is a severe infection in renal transplant recipients. Surgical debridement combined with antifungals, especially liposomal amphotericin B and posaconazole, can significantly improve patient's overall survival.
\end{abstract}

Keywords: Mucormycosis, Renal transplant recipient

\section{Background}

Mucormycosis has become an increasingly emerging lifethreatening invasive fungal infection especially in immunocompromised patients in the past decades, including patients with hematological malignancies and hematopoietic stem cell transplant recipients, solid organ transplant recipients, patients with diabetes mellitus, surgical patients, patients with burns, injection drug users, trauma patients, and those undergoing deferoxamine therapy [1-4]. The infection rates of post-transplant fungal infections were approximately 2-14\% [5]. Mucormycosis is associated with the longest duration of hospitalization and the shortest 2year survival in renal transplant (RT) patients, although it comprises only $2-6 \%$ of invasive fungal infection [6].

In order to identify the epidemiology, risk factors, prognosis of mucormycosis and outcome in renal transplant recipients, we examined all published cases of

\footnotetext{
* Correspondence: chenjianghua@zju.edu.cn

${ }^{1}$ Kidney Disease Center, The First Affiliated Hospital, College of Medicine, Zhejiang University, No. 79 Qingchun Road, Hangzhou 310003, Zhejiang, People's Republic of China

Full list of author information is available at the end of the article
}

mucormycosis in RT patients. We assessed the demographic data, clinical, histopathological and microbiological findings, laboratory tests, management and outcomes of the RT patients with mucormycosis.

\begin{abstract}
Methods
Literature search

Our goal was to discover the demographic characteristics, the underlying conditions, the site and pattern of infection, microbiologic and histopathologic findings, management and outcomes in RT patients with mucormycosis by review published cases. The literature search was limited to case or case series of mucormycosis in RT patients reported in English language. The PubMed database was searched for all mucormycosis case until December 2015 using the following key words: renal transplant, renal transplantation, kidney transplant, kidney transplantation, zygomycosis, mucormycosis, phycomycosis, Absidia (Mycocladus), Apophysomyces, Cokeromyces, Cunninghamella, Mucor, Rhizopus, Rhizomucor, Saksenaea, and Syncephalastrum.
\end{abstract}




\section{Case selection criteria}

Studies were eligible for inclusion if they reported a case or case series of mucormycosis in RT patients. Only cases which documented the following data were included in the review: age and sex of patients, anatomical location of infection, diagnostic methods, therapeutic strategy (including antifungal drugs and surgical therapy) and outcome. Zygomycosis caused by Entomophthorales was not included in this review.

\section{Data extraction}

From each case, we extracted publication year, sex and age of patients, primary underlying condition, duration from transplantation to disease onset, allograft rejection events and the primary site of infection at time of diagnosis, fungal culture, histopathology, antifungal therapy, surgical therapy and outcomes.

\section{Statistical analysis}

The association between potential risk factors and mortality were performed by using univariate analyses. Logistic regression analysis was used for multivariate analysis of variables found to be significant with univariate analysis. The variables were considered for inclusion in a multivariate model if they have a $p<0.20$ on univariate analysis. Values of 2-tailed $p<0.05$ were considered statistically significant. All statistical analyses were performed using the Statistics Package for Social Science (SPSS) version 17.0.

\section{Results}

Using our search strategy, it was yielded 253 articles reporting mucormycosis in RT patients. Abstracts and/or full texts were reviewed by two authors. One hundred and thirty-two articles were excluded because data of age, sex, therapy or outcome were not available. Entomophthoromycosis were exclude because the prognosis of diseases caused by Entomophthorales and Mucorales were different. Articles reported in non-English language were also excluded. Finally, a total of 174 cases of mucormycosis in renal transplant recipients were identified in 123 articles published from 1970 to 2015 (Additional file 1). The reporting of mucormycosis in renal transplant recipients was increasing during the study period (Fig. 1). One hundred and thirty-four cases were published after 2000, accounting for $77 \%$ of the patients (Fig. 1 ).

\section{Demography and medical history}

The demographic characteristics and underlying clinical conditions of the RT recipients with mucormycosis are summarized in Table 1 . The average age of the 174 patients was 45.9 years (range, 11-70 years), with male representing the majority $(76 \%)$. The most common underlying diseases was diabetes mellitus (43.1\%). Nine of them were type 1 diabetes, 6 were type 2 diabetes, and 10

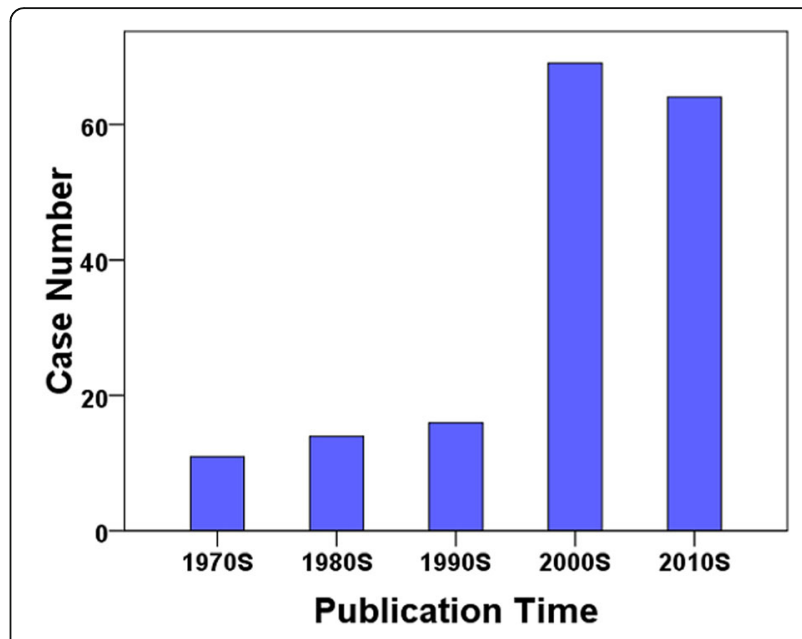

Fig. 1 Case numbers of mucormycosis since the 1970s

were post-transplant diabetes. In 50 of them the diabetes type was not described. The overall mortality of patients with diabetes mellitus was $33.3 \%$. Other underlying conditions including surgery, dental extraction, HIV infection, malignancy, trauma, and deferoxamine therapy. Of note, thirty $(25.1 \%)$ patients had no special medical history apart from RT. The median time duration from RT to establishing the diagnosis of mucormycosis was 2.5 months (range, 2 days-20 years).

One hundred and sixty-two patients received RT alone, the other 12 patients received combined solid organ transplantation, including the pancreas, liver, and heart. Although the mortality in combined organ transplantation recipients was higher than that in recipients received kidney transplantation alone $(75.0 \%$ vs $43.8 \%)$, combined transplantation was not a risk factor for mortality due to mucormycosis (unadjusted OR, 0.22; 95\% CI, 0.11-1.64). Acute allograft rejection occurred in 52(29.9\%) patients, $33(63.5 \%)$ of them received induction therapy. Neither anti-allograft rejection therapy nor induction therapy was risk factor of mortality due to mucormycosis $(p>0.20)$. The anti-rejection drugs were discontinued or reduced in $98(56.3 \%)$ patients.

\section{Sites of infection}

According to the clinical presentation and anatomic localization, there were 7 major clinical forms of mucormycosis infection: (1) rhinocerebral, (2) pulmonary, (3) cutaneous, (4) gastrointestinal, (5) graft kidney (6) disseminated, and (7) other uncommon sites (Table 2). Disseminated infection was defined as infection at 2 noncontiguous sites. The types of infection by site at the time of diagnosis in our review were shown in Table 2. Overall, rhinocerebral mucormycosis is the most common form (33.3\%), followed by pulmonary $(25.9 \%)$, disseminated $(14.4 \%)$, transplanted kidney $(11.5 \%)$, cutaneous $(7.5 \%)$, gastrointestinal $(5.7 \%)$, 
Table 1 Demographic and clinical characteristics of 174 renal transplant recipients with mucormycosis

\begin{tabular}{|c|c|c|}
\hline Feature & All patients & $\begin{array}{l}\text { Proportion of patients } \\
\text { who died }\end{array}$ \\
\hline Mean age, $y$ & 45.9 (range 11-70) & \\
\hline \multicolumn{3}{|l|}{ Gender } \\
\hline Male & 133(76\%) & $58 / 133(43.6 \%)$ \\
\hline Female & $41(24 \%)$ & 16/41 (39.0\%) \\
\hline \multicolumn{3}{|l|}{ Underlying conditions } \\
\hline No & $30 / 174(25.1 \%)$ & $15 / 30(50.0 \%)$ \\
\hline Diabetes & 75/174 (43.1\%) & 25/75 (33.3\%) \\
\hline Other conditions ${ }^{a}$ & 19/174 (3.4\%) & $7 / 19(36.8 \%)$ \\
\hline Not reported & $50 / 174(28.7 \%)$ & $27 / 50(54.0 \%)$ \\
\hline \multicolumn{3}{|l|}{ Transplanted organs } \\
\hline Kidney alone & 162/174(93.1\%) & $71 / 162(43.8 \%)$ \\
\hline $\begin{array}{l}\text { Kidney combined with } \\
\text { other organs }\end{array}$ & $12 / 174(6.9 \%)$ & 9/12(75.0\%) \\
\hline \multicolumn{3}{|c|}{ Anti-allograft rejection therapy } \\
\hline Yes & 33/174(18.97\%) & $12 / 33(36.4 \%)$ \\
\hline No & 16/174(9.1\%) & $7 / 16(43.8 \%)$ \\
\hline Not reported & 125/174(71.8\%) & $55 / 125(44.0 \%)$ \\
\hline \multicolumn{3}{|l|}{ Induction therapy } \\
\hline Yes & $33 / 174(19.0 \%)$ & 12/33(36.4\%) \\
\hline No & 16/174(9.2\%) & $7 / 16(43.8 \%)$ \\
\hline Not reported & 125/174(71.8\%) & $55 / 125(71.8 \%)$ \\
\hline \multicolumn{3}{|c|}{ Reduce immunosuppressive drugs } \\
\hline Yes & $98(56.3 \%)$ & 38/98(38.8\%) \\
\hline No & $6(3.4 \%)$ & $2 / 6(33.3 \%)$ \\
\hline Not reported & $70(40.2 \%)$ & $34 / 70(48.6 \%)$ \\
\hline \multicolumn{3}{|l|}{ Diagnostic methods } \\
\hline Histopathology only & 72/174(41.4\%) & $33 / 72(45.8 \%)$ \\
\hline Culture only & 25/174(14.4\%) & $9 / 25(36.0 \%)$ \\
\hline $\begin{array}{l}\text { Histopathology and } \\
\text { culture }\end{array}$ & 77/174(44.3\%) & $32 / 77(41.6 \%)$ \\
\hline
\end{tabular}

Including surgery, dental extraction, HIV infection, malignancy, trauma, and deferoxamine therapy

peritoneal $(1.1 \%)$ and artery stent $(0.6 \%)$. The mortality of mucormycosis in RT recipients was varied with the site of infection: $76 \%$ of patients with disseminated mucormycosis, $42.2 \%$ with pulmonary infection, and $55.6 \%$ with renal graft infection (Table 2).

Of the 58 patients with rhinocerebral infection, the initial symptoms of $33(56.9 \%)$ patients were headache and $25(43.1 \%)$ patients had a history of diabetes or hyperglycemia. The mortality of disseminated mucormycosis was much higher than that of localized diseases $(p<0.001$, OR $=5.41 ; 95 \% \mathrm{CI}, 2.04-14.37)$.

Signs that suggest graft kidney mucormycosis were nonspecific, including fever, abdominal pain, oliguria, and graft
Table 2 Infection patterns among 174 renal transplant recipients with mucormycosis, 74 of whom died

\begin{tabular}{lll}
\hline Type of infection & Proportion of all patients & $\begin{array}{l}\text { Number of patients who } \\
\text { died/total number (\%) }\end{array}$ \\
\hline Disseminated & $25(14.4 \%)$ & $19 / 25(76.0 \%)$ \\
Pulmonary & $45(25.9 \%)$ & $19 / 45(42.2 \%)$ \\
Gastrointestinal & $10(5.7 \%)$ & $4 / 10(40 \%)$ \\
Cutaneous & $13(7.5 \%)$ & $3 / 13(23.1 \%)$ \\
Graft kidney & $20(11.5 \%)$ & $11 / 20(55.6 \%)$ \\
Rhinocerebral & $58(33.3 \%)$ & $18 / 58(31.0 \%)$ \\
Peritoneal & $2(1.1 \%)$ & $0 / 2(0 \%)$ \\
Artery stent & $1(0.6 \%)$ & $0 / 1(0 \%)$ \\
Total & $174(100 \%)$ & $74 / 174(42.5 \%)$
\end{tabular}

dysfunction. However, absence of this findings should not exclude the possibility of mucormycosis. Fever was variable and may be absent in up to half of cases. There was only one case accompany with urinary leak before graft infection.

There were two cases of peritoneal dialysis-associated zygomycete peritonitis after renal transplantation. The patients have nonspecial symptoms, including abdominal pain and cloudy dialysis effluent. Tenckoff dialysis catheter were removed in the two zygomycete peritonitis patients and converted to maintain hemodialysis.

\section{Secular trends in reported hosts}

Our study finds that there was a rising incidence of mucormycosis in RT recipients in the past 5 decades (Fig. 1). The majority cases documented by culture were reported since the 2000s. This could be caused by the advances in diagnostic techniques.

\section{Microbiologic and histopathologic findings}

All patients were confirmed as mucormycosis by histological examination and/or fungal culture. Histological examination was performed in 160 cases. 149 (85.6\%) of which revealed typical hyphae. Of the 174 cases, 77 (44.3\%) were diagnosed by both histopathology and culture, $25(14.4 \%)$ by culture only, $72(41.4 \%)$ by histopathology only (Table 1). In 88 patients pathogenic zygomyectes were identified to species level (Table 3). The most frequent pathogen was Rhizopus species (59.1\%), followed by Mucor species (13.6\%) and Cunninghamella species (8.0\%).

\section{Sex and mucormycosis}

Most cases of mucormycosis in RT recipients (76\%) were male. Infection with Mycocladus (100\%), Apophysomyces elegans (80.0\%), Mucor (90.9\%) and Rhizopus rhizopodiformis $(100 \%)$ was highly associated with male gender (Table 4, Fig. 2). 
Table 3 Microbiological findings for 88 renal transplant recipients with mucormycosis, 38 of whom died

\begin{tabular}{lll}
\hline Isolated zygomycetes & $\begin{array}{l}\text { Number (\%) of } \\
\text { all patients }\end{array}$ & $\begin{array}{l}\text { Number of patients who } \\
\text { died/total number (\%) }\end{array}$ \\
\hline Mycocladus (Absidia) species & $4(4.5 \%)$ & $2 / 4(50 \%)$ \\
Apophysomyces elegans & $5(5.7 \%)$ & $3 / 5(60 \%)$ \\
Cunninghamella species & $7(8.0 \%)$ & $2 / 7(28.6 \%)$ \\
Mucor species & $12(13.6 \%)$ & $7 / 12(58.3 \%)$ \\
Rhizomucor species & $6(6.8 \%)$ & $3 / 6(50 \%)$ \\
Rhizopus species & $52(59.1 \%)$ & $19 / 52(36.5 \%)$ \\
$\quad$ Not speciated & $26(29.5 \%)$ & $7 / 26(26.9 \%)$ \\
Rhizopus oryzae & $18(20.5 \%)$ & $7 / 18(38.9 \%)$ \\
Rhizopus microsporus & $5(5.7 \%)$ & $4 / 5(80 \%)$ \\
Rhizopus rhizopodiformis & $3(3.4 \%)$ & $1 / 3(33.3 \%)$ \\
Other & $2(2.3 \%)$ & $0 / 2(0 \%)$ \\
Total & $88(100 \%)$ & $36 / 88(40.9 \%)$ \\
\hline
\end{tabular}

\section{Therapy}

The treatment strategies included antifungal therapy, surgery, and reduction of immunosuppression (Table 5). One hundred and twenty-one (69.5\%) patients received both antifungals and surgery therapy. The survival rate of this group patients $(70.2 \%)$ was much higher than those who received either antifungal therapy alone (32.4\%) or surgery alone $(36.4 \%)(p<0.001)$. Of the 174 cases reviewed, 155 (89\%) received antifungal chemotherapy (Table 5). The survival rate of patients received deoxycholate amphotericin B (37/78, 47.4\%), liposomal amphotericin B (47/64, 73.4\%) and posaconazole $(12 / 13,92.3 \%)$ therapy was higher than those receiving other antifungals $(4 / 19,21.1 \%)$ or no

Table 4 Relationship between gender and pathogenic fungal species in 174 renal transplant recipients with mucormycosis

\begin{tabular}{ll}
\hline Isolated zygomycetes & $\begin{array}{l}\text { Number of male cases/total } \\
\text { no. of cases (\%) }\end{array}$ \\
\hline Mycocladus (Absidia) species & $4 / 4(100 \%)$ \\
Apophysomyces elegans & $4 / 5(80.0 \%)$ \\
Cunninghamella species & $5 / 7(71.4 \%)$ \\
Mucor species & $10 / 11(90.9 \%)$ \\
Rhizomucor species & $3 / 6(50.0 \%)$ \\
Rhizopus species & $39 / 52(75.0 \%)$ \\
Not speciated & $19 / 26(73.1 \%)$ \\
Rhizopus oryzae & $14 / 18(77.8 \%)$ \\
Rhizopus microsporus & $3 / 5(60.0 \%)$ \\
Rhizopus rhizopodiformis & $3 / 3(100 \%)$ \\
Other & $1 / 2(50.0 \%)$ \\
Unidentified zygomycetes & $66 / 86(76.7 \%)$ \\
Total & $133 / 174(76.4 \%)$ \\
\hline
\end{tabular}

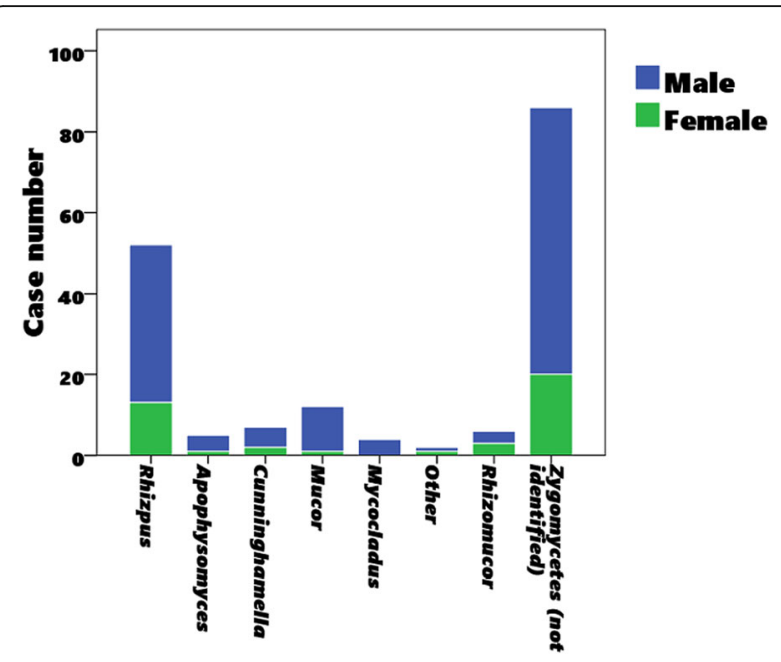

Fig. 2 Male to female ratio of pathogenic fungal species in 174 renal transplant recipients with mucormycosis

treatment $(0 / 8,0 \%),(p<0.001)$ (Table 6$)$. The overall survivals in patients receiving posaconazole and lipid amphoterincin $\mathrm{B}$ were higher than that receiving deoxycholate formulation (92.3\% and $73.4 \%$ vs $47.4 \%$ ) (Table 4 ).

\section{Discussion}

There have been several studies about mucormycosis in patients with diabetes mellitus, $[7,8]$ and hematologic malignancies $[9,10]$. Although there are some studies focus on the epidemiology and risk factors of mucormycosis in solid organ transplant recipients $[11,12]$, this is the first critical review of the epidemiology, diagnosis, and treatment of mucormycosis in RT patients. We find that the incidence of mucormycosis in RT patients was growing within the past several decades. The growing trend of mucormycosis may be associated with enhanced use of immunosuppressive therapy, prolonged prophylaxis with antifungals lacking

Table 5 Therapy of 174 renal transplant recipients with mucormycosis

\begin{tabular}{lll}
\hline Therapy & Patient number & Survived/total number \\
\hline Amphotericin B formulation & & \\
\multicolumn{1}{c}{ Deoxycholate } & $78 / 174(44.8 \%)$ & $37 / 78(47.4 \%)$ \\
$\quad$ Lipid & $64 / 174(36.8 \%)$ & $47 / 64(73.4 \%)$ \\
Posaconazole & $13 / 174(7.5 \%)$ & $12 / 13(92.3 \%)$ \\
Itraconazole, voriconazole, & $19 / 174(11.0 \%)$ & $4 / 19(21.1 \%)$ \\
fluconazole, encanocandins, & & \\
and no antifungal therapy & & \\
Surgery alone & $11 / 174(6.3 \%)$ & $4 / 11(36.4 \%)$ \\
Surgery and antifungal therapy & $121 / 174(69.5 \%)$ & $85 / 121(70.2 \%)$ \\
Antifungal alone & $34 / 174(19.5 \%)$ & $11 / 34(32.4 \%)$ \\
None & $8 / 174(4.6 \%)$ & $0 / 8(0 \%)$ \\
\hline
\end{tabular}


Table 6 Risk factors for mortality of renal transplant recipients with mucormycosis

\begin{tabular}{lll}
\hline Variable & OR $(95 \% \mathrm{Cl})$ & $P$ \\
\hline Extent of infection & & \\
Localized & Reference & \\
Disseminated & $5.41(2.04-14.37)$ & 0.001 \\
Organism & & \\
$\quad$ Rhizopus spp. & Reference & \\
Other organisms & & $>0.1$ \\
Diabetes & & \\
No & Reference & \\
$\quad$ Diabetes & $0.43(0.20-0.89)$ & 0.02 \\
Antifungal therapy & & \\
None & Reference & \\
Amphotericin B deoxycholate & $0.30(0.09-0.97)$ & 0.04 \\
Lipid amphotericin B & $0.10(0.03-0.33)$ & $<0.001$ \\
$\begin{array}{l}\text { Posaconazole alone and combined } \\
\text { with other antifungals }\end{array}$ & $0.02(0.00-0.22)$ & $<0.01$ \\
Surgery & & \\
Surgery as primary therapy & & \\
Without surgery therapy & Reference & \\
\hline
\end{tabular}

activity against zygomycetes, the rising prevalence of diabetes mellitus, advances in diagnostic techniques, increased awareness of clinicians and publication bias.

Our study indicates that there is a higher prevalence of mucormycosis in male RT patients. This finding is consistent with a comprehensive literature review of mucormycosis [3]. While we cannot find the predictor factors in male patients in our critical review. We postulate the sex preference may be owing to the protective role of estrogen, [13] habits and customs, or other differences between male and female.

Mucormycosis remains a seriously threat in patients with diabetes mellitus $[2,7]$. Our study also shows that RT patients with diabetes mellitus are prone to develop mucormycosis. However, diabetes mellitus is not an independent risk factor for mortality in RT patients with mucormycosis. This is mainly due to the good control of blood glucose in diabetes patients and the enhanced treatment with statins which are active against some zygomycetes [14, 15].

Although the overall mortality rate in patients received combined solid organ transplantation higher than that in patients received RT alone (75.0\% vs $43.8 \%)$, combined organ transplantation is not an independent risk factor for mortality $(p=0.22)$. The statistic indifference may be caused by the low number of patients received combined organ transplantation.

Theoretically the use of immunosuppressive therapeutics, especially the induction therapy and the antiallograft rejection therapy, are risk factors of fungal infection. Immunosuppressive agents can increase patient's susceptibility to mucormycosis by causing function defects of macrophages and neutrophils and/or by causing drug-induced diabetes [16]. However, we did not find that anti-allograft rejection therapy influences the incidence and the mortality of mucormycosis. This could be explained by the fact that the numbers of patients not receiving anti-allograft rejection treatment (16 patients, 9.1\%) and reducing immunosuppressants (6 patients, $3.4 \%)$ are very small. For some RT recipients, another reason for this indifferent is that mucormycosis occurred prior to immunosuppressive treatment.

Renal involvement is a rare manifestation of mucormycosis. In RT recipients, graft mucormycosis is relatively common (11.5\%). Among RT patients with localized mucormycosis, the mortality rate of graft kidney mucormycosis is the highest $(11 / 20,55.6 \%)$. They were suspected as acute rejection upon clinical signs and symptoms in $20 \%$ RT recipients. So, patients who are suspected as rejection should be alert for mucormycosis before anti-rejection therapy.

The overall survival in patients receiving amphotericin B combined with surgery therapy is higher than those receiving amphotericin B alone or surgery alone. Therefore, the first-line therapy for mucormycosis in RT recipients should be amphotericin B lipid formulation combined with surgery debridement. This is consent with the recommendation to general patients [3].

The overall survival in patients receiving lipid amphoterincin B is higher than that receiving deoxycholate formulation. Renal toxicity and is the most serious side effect of amphotericin B. We postulate that the lower overall survival of deoxycholate amphoterin B may be associated with its side effects, which hold back deoxycholate formuation to be used. Posaconazole was a new azole antifungal with activity against zygomycetes. The overall survival is 92\% in patients receiving posaconazole therapy, its higher than those receiving amphotericin B. Recent studies also have shown overall success rates of $60-70 \%$ with posaconazole as salvage therapy for zygomycetes infection [17, 18]. These encouraging data suggest that posaconazole may represent a prospective drug against mucormycosis.

\section{Conclusion}

In conclusion, mucormycosis is a fungal infection with high mortality in RT patients. In RT recipients with disseminated and graft kidney mucormycosis have the worst prognosis. Surgical debridement combined with antifungals (amphotericin B formulation and posaconazole) can significantly improve patient's overall survival. The effect of liposomal amphotericin B and posaconazole seems better than amphotericin B deoxycholate against mucormycosis in renal transplant recipients. Clinicians should increase precautions to mucormycosis in RT recipients. 


\section{Additional file}

Additional file 1: Published cases of mucormycosis in renal transplant recipients included in this review. (DOCX $105 \mathrm{~kb}$ )

\section{Abbreviation}

RT: Renal transplantation

\section{Acknowledgements}

Not applicable.

\section{Funding}

This work was supported by funds from the National Natural Science Foundation of China, to JQ (Grant no. 81571969) and to HY (Grant no. 81300619).

\section{Availability of data and materials}

We declare that the data supporting the conclusions of this article are fully described within the article.

\section{Authors' contributions}

YS and JC designed the study and drafted an outline. YS, JQ, GG, GL, HY and JW participated in data analysis. YS and JQ draft of initial manuscript. YS, JQ, GG, GL, HY, JW and JC approved the final content of this manuscript.

\section{Competing interests}

The authors declare that they have no competing interests.

\section{Consent for publication}

Not applicable.

\section{Ethics approval and consent to participate}

We have analyzed cases obtained from studies that were published.

Therefore, this study did not require approval from ethical committee.

\section{Publisher's note}

Springer Nature remains neutral with regard to jurisdictional claims in published maps and institutional affiliations.

\section{Author details}

${ }^{1}$ Kidney Disease Center, The First Affiliated Hospital, College of Medicine, Zhejiang University, No. 79 Qingchun Road, Hangzhou 310003, Zhejiang, People's Republic of China. ${ }^{2}$ Department of Dermatology, The First Affiliated Hospital, College of Medicine, Zhejiang University, Hangzhou, People's Republic of China. ${ }^{3}$ Azienda Ospedaliero-Universitaria Ospedali Riuniti, Ancona, Italy.

Received: 12 December 2016 Accepted: 4 April 2017

Published online: 18 April 2017

\section{References}

1. Lewis RE, Kontoyiannis DP. Epidemiology and treatment of mucormycosis. Future Microbiol. 2013;8(9):1163-75

2. Binder U, Maurer E, Lass-Florl C. Mucormycosis-from the pathogens to the disease. Clin Microbiol Infect. 2014;20(Suppl 6):60-6.

3. Roden MM, Zaoutis TE, Buchanan WL, Knudsen TA, Sarkisova TA, Schaufele RL, Sein M, Sein T, Chiou CC, Chu JH, et al. Epidemiology and outcome of zygomycosis: a review of 929 reported cases. Clin Infect Dis. 2005;41(5):634-53.

4. Lanternier F, Sun HY, Ribaud P, Singh N, Kontoyiannis DP, Lortholary O. Mucormycosis in organ and stem cell transplant recipients. Clin Infect Dis. 2012;54(11):1629-36.

5. Gandhi BV, Bahadur MM, Dodeja H, Aggrwal V, Thamba A, Mali M. Systemic fungal infections in renal diseases. J Postgrad Med. 2005;51(Suppl 1):S30-6.

6. Kontoyiannis DP, Marr KA, Park BJ, Alexander BD, Anaissie EJ, Walsh TJ, Ito J, Andes DR, Baddley JW, Brown JM, et al. Prospective surveillance for invasive fungal infections in hematopoietic stem cell transplant recipients, 2001 2006: overview of the transplant-associated infection surveillance network (TRANSNET) database. Clin Infect Dis. 2010;50(8):1091-100.

7. Lanternier F, Lortholary O. Zygomycosis and diabetes mellitus. Clin Microbiol Infect. 2009;15(Suppl 5):21-5.
8. Rammaert B, Lanternier F, Poiree S, Kania R, Lortholary O. Diabetes and mucormycosis: a complex interplay. Diabetes Metab. 2012;38(3):193-204.

9. Kontoyiannis DP, Wessel VC, Bodey GP, Rolston KV. Zygomycosis in the 1990s in a tertiary-care cancer center. Clin Infect Dis. 2000;30(6):851-6.

10. Kontoyiannis DP, Lionakis MS, Lewis RE, Chamilos G, Healy M, Perego C, Safdar A, Kantarjian H, Champlin R, Walsh TJ, et al. Zygomycosis in a tertiary-care cancer center in the era of Aspergillus-active antifungal therapy: a case-control observational study of 27 recent cases. J Infect Dis. 2005;191(8):1350-60.

11. Singh N, Aguado JM, Bonatti H, Forrest G, Gupta KL, Safdar N, John GT, Pursell KJ, Munoz P, Patel R, et al. Zygomycosis in solid organ transplant recipients: a prospective, matched case-control study to assess risks for disease and outcome. J Infect Dis. 2009;200(6):1002-11.

12. Cuenca-Estrella M, Bernal-Martinez L, Isla G, Gomez-Lopez A, Alcazar-Fuoli L, Buitrago MJ. Incidence of zygomycosis in transplant recipients. Clin Microbiol Infect. 2009:15(Suppl 5):37-40.

13. Restrepo A, Salazar ME, Cano LE, Stover EP, Feldman D, Stevens DA Estrogens inhibit mycelium-to-yeast transformation in the fungus Paracoccidioides brasiliensis: implications for resistance of females to paracoccidioidomycosis. Infect Immun. 1984;46(2):346-53.

14. Bellanger AP, Tatara AM, Shirazi F, Gebremariam T, Albert ND, Lewis RE, Ibrahim AS, Kontoyiannis DP. Statin concentrations below the minimum inhibitory concentration attenuate the virulence of Rhizopus oryzae. J Infect Dis. 2016;214(1):114-21.

15. Kontoyiannis DP. Decrease in the number of reported cases of zygomycosis among patients with diabetes mellitus: a hypothesis. Clin Infect Dis. 2007:44(8):1089-90.

16. Pagano L, Valentini CG, Fianchi L, Caira M. The role of neutrophils in the development and outcome of zygomycosis in haematological patients. Clin Microbiol Infect. 2009;15(Suppl 5):33-6.

17. Riley TT, Muzny CA, Swiatlo E, Legendre DP. Breaking the mold: a review of Mucormycosis and current pharmacological treatment options. Ann Pharmacother. 2016;50(9):747-57

18. van Burik JA, Hare RS, Solomon HF, Corrado ML, Kontoyiannis DP. Posaconazole is effective as salvage therapy in zygomycosis: a retrospective summary of 91 cases. Clin Infect Dis. 2006:42(7):e61-5.

\section{Submit your next manuscript to BioMed Central and we will help you at every step:}

- We accept pre-submission inquiries

- Our selector tool helps you to find the most relevant journal

- We provide round the clock customer support

- Convenient online submission

- Thorough peer review

- Inclusion in PubMed and all major indexing services

- Maximum visibility for your research

Submit your manuscript at www.biomedcentral.com/submit
Biomed Central 